\title{
Zonotopic Set-Membership State Estimation for Discrete-Time Descriptor LPV Systems
}

\author{
Ye Wang, Zhenhua Wang, Vicenç Puig and Gabriela Cembrano
}

\begin{abstract}
This technical note proposes a novel set-membership state estimation approach based on zonotopes for discrete-time descriptor linear parameter-varying (LPV) systems. The consistency test between the system model and measured outputs is implemented to construct a parameterized intersection zonotope with respect to a correction matrix. With a defined zonotope minimization criterion, we propose a novel offline optimization problem to obtain the optimal correction matrix. In addition, with the proposed approach, an adaptive bound of the radius of the intersection zonotope is also provided. Finally, a case study with a truck-trailer system is shown to illustrate the proposed approach.
\end{abstract}

Index Terms-Set-membership approach, state estimation, zonotopes, descriptor systems, discrete-time LPV models.

\section{INTRODUCTION}

Set-membership approaches, known as a class of deterministic methods assuming an unknown-but-bounded description of uncertainties, have been widely investigated in the last decade. A direct application of a set-membership approach is state estimation [1], [2], where uncertainties originally bounded in some given sets are propagated to system states using a mathematical model with measurement information. Besides, set-membership approaches have also been applied to parameter estimation [3], [4], fault diagnosis [5], [6] as well as fault-tolerant control [7] allowing a worst-case analysis.

In the literature, there are two categories of set-based estimation approaches: the one based on the interval observer and the other using a set-membership approach. The interval observer approach (see, e.g. [8], [9]) relies on separately generating upper and lower bounds of estimated states using two observers. However, it is usually non-trivial to design an interval observer since the design conditions are restrictive. Unlike this approach, a set-membership state estimation approach (e.g. [1]) makes use of a predefined geometrical set for uncertainty propagations. On the one hand, a zonotopic set-membership approach is able to obtain accurate estimation results taking into account that operations on zonotopes can

This work was partially funded by the Spanish State Research Agency (AEI) and the European Regional Development Fund (ERFD) through the projects DEOCS (ref. DPI2016-76493-C3-3-R) and SCAV (ref. DPI2017-88403-R), the FPI grant (ref. BES-2014-068319). Z. Wang was supported by National Natural Science Foundation of China (Grant No. 61273162, 61403104).

Y. Wang, V. Puig and G. Cembrano are with Advanced Control Systems (SAC) Research Group at Institut de Robòtica i Informàtica Industrial, CSIC-UPC, Universitat Politècnica de Catalunya-BarcelonaTech (UPC), C/ Llorens i Artigas 4-6, 08028 Barcelona, Spain (e-mail: ywang@iri.upc.edu, vicenc.puig@upc.edu, cembrano@iri.upc.edu)

Z. Wang is with School of Astronautics, Harbin Institute of Technology, Harbin 150001, P.R. China (e-mail:zhenhua.wang@ hit.edu.cn)

G. Cembrano is also with Cetaqua, Water Technology Centre, Ctra. d'Esplugues 75, Cornellà de Llobregat, 08940 Barcelona, Spain effectively eliminate wrapping effects inherent to the use of an iterative approach [10]. On the other hand, the computation load of this approach is low, since the computation of linear transformations (i.e. Minkowski sum) is efficient. As also discussed in [11], the complexity of operations on zonotopes can be significantly reduced by means of a suitable zonotope order reduction technique. Besides, instead of using online computations as in [2], an offline method could be used for the design of parameters in this approach.

In a large amount of industrial processes, system behaviors are described including not only system dynamics by differential/difference equations but also static relations by algebraic equations due to energy or mass balances. Such systems are called descriptor systems, also known as singular [12] or differential-algebraic systems [13], which have played a significant role in a variety of applications, such as water distribution networks [14], aircraft systems [15] and chemical systems [16]. In [17], the reachability analysis of nonlinear descriptor systems has been investigated based on the setbased approach to propagate a zonotopic reachable set. A nonlinear descriptor model can be formulated as a polytopic linear parameter-varying (LPV) model (see, e.g. [18], [19]). However, few research work in the literature related to setmembership state estimation approaches can be found for this type of systems.

The main contribution of this note is to propose a zonotopic set-membership state estimation approach for discrete-time descriptor LPV systems affected by unknown-but-bounded system disturbances and measurement noise. The consistency test between the system model and measured outputs is implemented by approximating the consistent state set in a parameterized intersection zonotope. A novel zonotope minimization criterion is defined, which is used for an offline optimization problem to obtain polytopic correction matrices. With the proposed approach, an adaptive bound of the radius of the intersection zonotope is provided. Finally, the proposed approach is tested through a case study of the truck-trailer system inspired by [19].

Notation: For $z \in \mathbb{R}^{n}$, the 2-norm is denoted by $\|z\|=$ $\sqrt{z^{\top} z}$, the weighted 2-norm is denoted by $\|z\|_{P}=\sqrt{z^{\top} P z}$, where $P \in \mathbb{R}^{n \times n}$ with $P=P^{\top} \succ 0$ is a weighting matrix, and $\operatorname{diag}(z)$ denotes a diagonal matrix with the diagonal elements determined by $z$. For $z_{k} \in \mathbb{R}^{n}, \forall k \in \mathbb{N}$, the $\mathcal{L}_{\infty}$ norm (peak norm) is denoted by $\|z\|_{\infty}=\sup _{k}\left\|z_{k}\right\|$. We use $\star$ to represent a term that can be induced by symmetry. For a matrix $M, M$ is said to be positive definite if the scalar $z^{\top} M z$ is positive for arbitrary non-zero column vector $z$ 
of real numbers, which is denoted by $M \succ 0$ while $M \prec 0$ denotes negative definiteness. $M \succeq 0$ denotes positive semidefiniteness and $M \preceq 0$ denotes negative semi-definiteness. Besides, $\operatorname{rank}(M)$ returns the rank of $M$. We use $I_{r}$ to denote an identity matrix of dimension $r$. Note that we may drop the index when the dimension can be inferred in the context.

A $r$-order zonotope $\mathcal{Z}=\langle p, H\rangle \in \mathbb{R}^{n}(n \leq r)$ with $2^{r}$ vertices is defined as

$$
\mathcal{Z}=\langle p, H\rangle=\left\{p+H z,\|z\|_{\infty} \leq 1\right\},
$$

with the center $p \in \mathbb{R}^{n}$ and a generator matrix $H \in \mathbb{R}^{n \times r}$. Let us denote $\mathbf{B}^{r}=[-1,+1]^{r}$ as a hypercube of the order $r$ and the Minkowski sum as $\oplus$. Therefore, the zonotope $\mathcal{Z}=$ $\langle p, H\rangle$ can be also formulated as $\mathcal{Z}=p \oplus H \mathbf{B}^{r}$. Besides, the following properties hold

$$
\begin{aligned}
\left\langle p_{1}, H_{1}\right\rangle \oplus\left\langle p_{2}, H_{2}\right\rangle & =\left\langle p_{1}+p_{2},\left[\begin{array}{ll}
H_{1} & H_{2}
\end{array}\right]\right\rangle, \\
L \odot\langle p, H\rangle & =\langle L p, L H\rangle, \\
\langle p, H\rangle & \subseteq\left\langle p, \downarrow_{q, W}(H)\right\rangle \subseteq\langle p, \operatorname{rs}(H)\rangle,
\end{aligned}
$$

where $\odot$ denotes the linear image product, $L$ is a matrix of appropriate dimensions, $\downarrow_{q, W}(H)$ denotes the reduction operator with $n \leq q \leq r$ proposed in [2], $q$ is the maximum column of the generator matrix after reduction, $W$ is a weighting matrix of appropriate dimension, and $r s(H)$ denotes the row sum of the matrix $H$. The set $\langle p, r s(H)\rangle$ is also called the interval hull of $\mathcal{Z}$. Besides, we use $\mathcal{X} \times \mathcal{Y}$ to denote the Cartesian product of two sets $\mathcal{X}$ and $\mathcal{Y}$.

\section{Problem Statement and PReliminaries}

Consider the class of the discrete-time descriptor LPV systems described by

$$
\begin{aligned}
E x_{k+1} & =A\left(\theta_{k}\right) x_{k}+B\left(\theta_{k}\right) u_{k}+D\left(\theta_{k}\right) d_{k}, \\
y_{k} & =C x_{k}+F d_{k},
\end{aligned}
$$

where $x \in \mathbb{R}^{n_{x}}, u \in \mathbb{R}^{n_{u}}$ and $y \in \mathbb{R}^{n_{y}}$ denote the state, input and output vectors, respectively. $d \in \mathbb{R}^{n_{d}}$ denotes the system uncertainty vector. $C \in \mathbb{R}^{n_{y} \times n_{x}}$ and $F \in \mathbb{R}^{n_{y} \times n_{d}}$ are measurement matrices. Besides, $E \in \mathbb{R}^{n_{x} \times n_{x}}$ is a singular matrix corresponding to the definition of the descriptor system. As in [20], $A\left(\theta_{k}\right), B\left(\theta_{k}\right)$ and $D\left(\theta_{k}\right)$ are matrices taking the following polytopic form: $A\left(\theta_{k}\right)=\sum_{j=1}^{h} \rho_{j}\left(\theta_{k}\right) A_{j}$, $B\left(\theta_{k}\right)=\sum_{j=1}^{h} \rho_{j}\left(\theta_{k}\right) B_{j}, D\left(\theta_{k}\right)=\sum_{j=1}^{h} \rho_{j}\left(\theta_{k}\right) D_{j}$, where $A_{j} \in \mathbb{R}^{n_{x} \times n_{x}}, B_{j} \in \mathbb{R}^{n_{x} \times n_{u}}, D_{j} \in \mathbb{R}^{n_{x} \times n_{d}}$ for $j=1, \ldots, h$ are known constant matrices. $\theta_{k} \in \mathbb{R}^{n_{\theta}}$ is a scheduling vector that can be measured online and $\rho_{j}\left(\theta_{k}\right)$, for $j=1, \ldots, h$ are weighting functions satisfying

$$
\rho_{j}\left(\theta_{k}\right) \geq 0, \sum_{j=1}^{h} \rho_{j}\left(\theta_{k}\right)=1, \forall j=1, \ldots, h .
$$

In (1), the uncertainty vector $d$ can also be divided as $d \triangleq$ $\left[w^{\top} v^{\top}\right]^{\top} \in \mathbb{R}^{n_{d}}$ with $n_{d}=n_{w}+n_{v}$, where $w \in \mathbb{R}^{n_{w}}$ and $v \in \mathbb{R}^{n_{v}}$ are the vectors of system disturbances and measurement noise. Besides, $D\left(\theta_{k}\right)=\left[\begin{array}{ll}\bar{D}\left(\theta_{k}\right) & 0\end{array}\right], F=\left[\begin{array}{ll}0 & \bar{F}\end{array}\right]$ with $\bar{D}\left(\theta_{k}\right) \in \mathbb{R}^{n_{x} \times n_{w}}$ and $\bar{F} \in \mathbb{R}^{n_{y} \times n_{v}}$.
Assumption 1. The uncertainty vector $d_{k}$ is unknown but bounded in a known centered zonotope $\mathcal{D}$ as $d_{k} \in \mathcal{D}=$ $\left\langle 0, H_{d}\right\rangle, \forall k \in \mathbb{N}$ and the initial uncertain state $x_{0}$ is also bounded in the zonotope $\mathcal{X}_{0}=\left\langle p_{0}, H_{0}\right\rangle$.

Remark 1. Since $d_{k} \in \mathcal{D}, \forall k \in \mathbb{N}$, the worst-case $d_{k}$ on the boundary of $\mathcal{D}=\left\langle 0, H_{d}\right\rangle$ is given by $\max _{b \in \mathbf{B}^{n_{d}}}\left\|H_{d} b\right\|$. Meanwhile, by definition, the $\mathcal{L}_{\infty}$ norm of $d_{k}$ is denoted by $\|d\|_{\infty}=\sup _{k}\left\|d_{k}\right\|$ with $k \in \mathbb{N}$, which satisfies

$$
\|d\|_{\infty}=\sup _{k}\left\|d_{k}\right\|=\max _{b \in \mathbf{B}^{n_{d}}}\left\|H_{d} b\right\| .
$$

Definition 1 (C-Observability [12], [13]). The descriptor LPV system (1) is said to be (completely) observable or $C$ observable if the initial condition $x_{0}$ of the system can be uniquely determined by $u_{k}$ and $y_{k}, \forall k \in \mathbb{N}$.

From Definition 1, refer to [13, Theorem 4.11] and [21], the following assumption is used in this note.

Assumption 2. The descriptor LPV system (1) is assumed to be $C$-observable. Then, matrices $E, A_{j}$ and $C$ satisfy $\operatorname{rank}\left[\begin{array}{c}z E-A_{j} \\ C\end{array}\right]=n_{x}, \forall j=1, \ldots, h, \forall z \in \mathbb{C}, z$ finite and $\operatorname{rank}\left[\begin{array}{c}E \\ C\end{array}\right]=n_{x}$

Lemma 1 ([19]). Since rank $\left[\begin{array}{l}E \\ C\end{array}\right]=n_{x}$ holds from Assumption 2, there exist two matrices $T$ and $N$ such that

$$
T E+N C=I_{n_{x}} .
$$

Then, the general solutions of $T$ and $N$ are given by

$$
\begin{aligned}
& T=\Psi^{\dagger} \alpha_{1}+S\left(I_{n_{x}+n_{y}}-\Psi \Psi^{\dagger}\right) \alpha_{1}, \\
& N=\Psi^{\dagger} \alpha_{2}+S\left(I_{n_{x}+n_{y}}-\Psi \Psi^{\dagger}\right) \alpha_{2},
\end{aligned}
$$

with $\Psi=\left[\begin{array}{c}E \\ C\end{array}\right], \alpha_{1}=\left[\begin{array}{c}I_{n_{x}} \\ 0\end{array}\right]$ and $\alpha_{2}=\left[\begin{array}{c}0 \\ I_{n_{y}}\end{array}\right]$, where $\Psi^{\dagger}$ is the pseudo-inverse matrix of $\Psi$ and $S$ is an arbitrary matrix of appropriate dimension.

According to the guaranteed state estimation approach in [1], since the uncertain state $x_{k-1}$ is bounded in the zonotope $\mathcal{X}_{k-1}=\left\langle p_{k-1}, H_{k-1}\right\rangle$ as a prior, the estimated uncertain state $x_{k}$ is over-approximated by implementing three steps including prediction, measurement and correction. We first introduce some necessary sets as follows.

Definition 2. Given the descriptor LPV system (1) and Assumption 1 holds, the uncertain state set $\overline{\mathcal{X}}_{k}$ propagated by (1a) is defined as

$$
\begin{aligned}
\overline{\mathcal{X}}_{k}=\left\{x_{k} \in \mathbb{R}^{n_{x}}\right. & \mid\left(E x_{k}-B\left(\theta_{k-1}\right) u_{k-1}\right) \\
& \left.\in\left(A\left(\theta_{k-1}\right) \mathcal{X}_{k-1} \oplus D\left(\theta_{k-1}\right) \mathcal{D}\right)\right\} .
\end{aligned}
$$

Definition 3. Given the descriptor LPV system (1) and a measured output $y_{k}$, the measurement consistent state set at time instant $k$ is defined as

$$
\mathcal{X}_{y_{k}}=\left\{x_{k} \in \mathbb{R}^{n_{x}} \mid\left(y_{k}-C x_{k}\right) \in F \mathcal{D}\right\} .
$$


Definition 4. Given the descriptor LPV system (1), the exact consistent uncertain state set $\mathcal{X}_{k}$, that encloses uncertain states consistent with measured outputs, is defined as

$$
\mathcal{X}_{k}=\overline{\mathcal{X}}_{k} \cap \mathcal{X}_{y_{k}} .
$$

In general, the steps of the set-membership state estimation approach are expressed as follows:

1) Prediction Step: Compute the uncertain state set $\overline{\mathcal{X}}_{k}$ at time instant $k$ using (1);

2) Measurement Step: Compute the measurement consistent state set $\mathcal{X}_{y_{k}}$ with the measured output $y_{k}$;

3) Correction Step: Compute a zonotopic outer approximation $\hat{\mathcal{X}}_{k}$ of the consistent uncertain state set $\mathcal{X}_{k}$ as an intersection between $\overline{\mathcal{X}}_{k}$ and $\mathcal{X}_{y_{k}}$.

In this note, the objective is to propose a set-membership state estimation approach for the descriptor LPV system (1), where all uncertain states are bounded by means of zonotopes. Note that it is impossible to find an explicit characterization of the exact consistent uncertain state set. By following the iterative steps presented above, the state bounding zonotope is defined as an outer-approximation of the exact consistent uncertain state set.

\section{MAIN Result}

We now present the set-membership state estimation approach for discrete-time descriptor LPV systems. Based on the system model (1), the prediction step can be implemented using the Minkowski sum and the model information through the forward set propagation. With the output data measured from the real system, the set defined in (7) can be obtained in the measurement step. Then, we compute the consistent state set (8) by a suitable approximation allowing to implement the consistency test in the correction step.

\section{A. Parameterized Intersection Zonotope}

The set $\mathcal{X}_{k}$ defined in (8) is a polytope obtained by an intersection between the zonotope $\overline{\mathcal{X}}_{k}$ and the polytope $\mathcal{X}_{y_{k}}$. To implement the steps of the set-membership state estimation approach in an iterative way, we first construct a parameterized intersection zonotope to over-approximate $\mathcal{X}_{k}$ in the following theorem, which includes the three steps of the set-membership state estimation.

Theorem 1. Consider the descriptor LPV system (1), $x_{k-1} \in$ $\hat{\mathcal{X}}_{k-1}=\left\langle\hat{p}_{k-1}, \hat{H}_{k-1}\right\rangle$, a measured output $y_{k}$, a measurement consistent state set $\mathcal{X}_{y_{k}}$ and a parameter-varying correction matrix $\Lambda\left(\theta_{k-1}\right) \in \mathbb{R}^{n_{x} \times n_{y}}$. Then, the consistent uncertain state set $\mathcal{X}_{k}$ is over-approximated by the zonotope $\hat{\mathcal{X}}_{k}\left(\Lambda\left(\theta_{k-1}\right)\right)$ :

$$
\begin{aligned}
\overline{\mathcal{X}}_{k} \cap \mathcal{X}_{y_{k}} & \subseteq \hat{\mathcal{X}}_{k}\left(\Lambda\left(\theta_{k-1}\right)\right) \\
& =\left\langle\hat{p}_{k}\left(\Lambda\left(\theta_{k-1}\right)\right), \hat{H}_{k}\left(\Lambda\left(\theta_{k-1}\right)\right)\right\rangle,
\end{aligned}
$$

with

$$
\begin{aligned}
\hat{p}_{k}\left(\Lambda\left(\theta_{k-1}\right)\right)= & \left(I-\Lambda\left(\theta_{k-1}\right) C\right) T A\left(\theta_{k-1}\right) \hat{p}_{k-1} \\
& +\left(I-\Lambda\left(\theta_{k-1}\right) C\right) T B\left(\theta_{k-1}\right) u_{k-1} \\
& +\left(N-\Lambda\left(\theta_{k-1}\right) C N+\Lambda\left(\theta_{k-1}\right)\right) y_{k}, \\
\hat{H}_{k}\left(\Lambda\left(\theta_{k-1}\right)\right)= & {\left[\begin{array}{lll}
\left(I-\Lambda\left(\theta_{k-1}\right) C\right) R_{k} & \Lambda\left(\theta_{k-1}\right) F H_{d}
\end{array}\right], \quad(10 \mathrm{~b}) } \\
R_{k}= & {\left[\begin{array}{lll}
T A\left(\theta_{k-1}\right) \hat{H}_{k-1} & T D\left(\theta_{k-1}\right) H_{d} & N F H_{d}
\end{array}\right] . }
\end{aligned}
$$

Proof. For any $\hat{x}_{k}$ satisfying $\hat{x}_{k} \in \overline{\mathcal{X}}_{k} \cap \mathcal{X}_{y_{k}}$, it implies $\hat{x}_{k} \in$ $\overline{\mathcal{X}}_{k}$ and $\hat{x}_{k} \in \mathcal{X}_{y_{k}}$. First, in the prediction step, from $\hat{x}_{k} \in \overline{\mathcal{X}}_{k}$ and (1a), there exists an unitary vector $z_{1}$ such that

$$
\begin{aligned}
E \hat{x}_{k}= & A\left(\theta_{k-1}\right) \hat{p}_{k-1}+B\left(\theta_{k-1}\right) u_{k-1} \\
& +\left[\begin{array}{ll}
A\left(\theta_{k-1}\right) \hat{H}_{k-1} & D\left(\theta_{k-1}\right) H_{d}
\end{array}\right] z_{1} .
\end{aligned}
$$

Therefore, in the measurement step, from $\hat{x}_{k} \in \mathcal{X}_{y_{k}}$ and (1b), there exists another unitary vector $z_{2}$ such that

$$
C \hat{x}_{k}-y_{k}=F H_{d} z_{2} .
$$

With a pair of $T$ and $N$ satisfying (4), (11) and (12) can be combined to obtain

$$
\begin{aligned}
\hat{x}_{k}= & T A\left(\theta_{k-1}\right) \hat{p}_{k-1}+T B\left(\theta_{k-1}\right) u_{k-1}+N y_{k} \\
& +\left[\begin{array}{lll}
T A\left(\theta_{k-1}\right) \hat{H}_{k-1} & T D\left(\theta_{k-1}\right) H_{d} & N F H_{d}
\end{array}\right]\left[\begin{array}{l}
z_{1} \\
z_{2}
\end{array}\right] .
\end{aligned}
$$

Set $R_{k}$ in (10c) and $s=\left[\begin{array}{ll}z_{1}^{\top} & z_{2}^{\top}\end{array}\right]^{\top}$. Finally, in the correction step, we introduce a parameter-varying correction ma$\operatorname{trix} \Lambda\left(\theta_{k-1}\right) \in \mathbb{R}^{n_{x} \times n_{y}}$ and a correction term $\Lambda\left(\theta_{k-1}\right) C R_{k} s$ such that substituting $\hat{x}_{k}$ in (12) by (13), it becomes

$$
\begin{aligned}
C R_{k} s= & (I-C N) y_{k}-C T A\left(\theta_{k-1}\right) \hat{p}_{k-1} \\
& -C T B\left(\theta_{k-1}\right) u_{k-1}+F H_{d} z_{2} .
\end{aligned}
$$

Adding and subtracting this correction term $\Lambda\left(\theta_{k-1}\right) C R_{k} s$ in (13), we have

$$
\begin{aligned}
\hat{x}_{k}= & T A\left(\theta_{k-1}\right) \hat{p}_{k-1}+T B\left(\theta_{k-1}\right) u_{k-1}+N y_{k} \\
& +\Lambda\left(\theta_{k-1}\right) C R_{k} s+\left(I-\Lambda\left(\theta_{k-1}\right) C\right) R_{k} s \\
= & T A\left(\theta_{k-1}\right) \hat{p}_{k-1}+T B\left(\theta_{k-1}\right) u_{k-1}+N y_{k} \\
& +\Lambda\left(\theta_{k-1}\right)(I-C N) y_{k}-\Lambda\left(\theta_{k-1}\right) C T A\left(\theta_{k-1}\right) \hat{p}_{k-1} \\
& -\Lambda\left(\theta_{k-1}\right) C T B\left(\theta_{k-1}\right) u_{k-1}+\left(I-\Lambda\left(\theta_{k-1}\right) C\right) R_{k} s \\
& +\Lambda\left(\theta_{k-1}\right) F H_{d} z_{2} \\
= & \left(I-\Lambda\left(\theta_{k-1}\right) C\right)\left(T A\left(\theta_{k-1}\right) \hat{p}_{k-1}+T B\left(\theta_{k-1}\right) u_{k-1}\right) \\
& +\left(N-\Lambda\left(\theta_{k-1}\right) C N+\Lambda\left(\theta_{k-1}\right)\right) y_{k} \\
& +\left[\left(I-\Lambda\left(\theta_{k-1}\right) C\right) R_{k} \quad \Lambda\left(\theta_{k-1}\right) F H_{d}\right]\left[\begin{array}{c}
s \\
z_{2}
\end{array}\right],
\end{aligned}
$$

from which we obtain the zonotope $\hat{\mathcal{X}}_{k}\left(\Lambda\left(\theta_{k-1}\right)\right)$ with the center $\hat{p}_{k}\left(\Lambda\left(\theta_{k-1}\right)\right)$ and the generator matrix $\hat{H}_{k}\left(\Lambda\left(\theta_{k-1}\right)\right)$ as in (10).

Remark 2. Along an iterative estimation procedure, the order of $\hat{\mathcal{X}}_{k}\left(\Lambda\left(\theta_{k-1}\right)\right), \forall k \in \mathbb{N}_{+}$is growing because at each time step, the term $\Lambda\left(\theta_{k-1}\right) F H_{d}$ is added into $\hat{H}_{k}\left(\Lambda\left(\theta_{k-1}\right)\right)$, $\forall k \in \mathbb{N}_{+}$. From the application point of view, the order of the 


$$
\begin{aligned}
\hat{p}_{k}\left(\Lambda\left(\theta_{k-1}\right)\right)= & \sum_{i=1}^{h} \sum_{j=1}^{h} \rho_{i}\left(\theta_{k-1}\right) \rho_{j}\left(\theta_{k-1}\right)\left(\left(I-\Lambda_{i} C\right) T A_{j} \hat{p}_{k-1}+\left(I-\Lambda_{i} C\right) T B_{j} u_{k-1}\right)+\sum_{i=1}^{h} \rho_{i}\left(\theta_{k-1}\right)\left(\left(N-\Lambda_{i} C N+\Lambda_{i}\right) y_{k}\right), \\
\hat{H}_{k}\left(\Lambda_{i}\left(\theta_{k-1}\right)\right)= & {\left[\sum_{i=1}^{h} \sum_{j=1}^{h} \rho_{i}\left(\theta_{k-1}\right) \rho_{j}\left(\theta_{k-1}\right)\left(\left(I-\Lambda_{i} C\right) T A_{j} \bar{H}_{k-1}\right) \quad \sum_{i=1}^{h} \sum_{j=1}^{h} \rho_{i}\left(\theta_{k-1}\right) \rho_{j}\left(\theta_{k-1}\right)\left(\left(I-\Lambda_{i} C\right) T D_{j} H_{d}\right)\right.} \\
& \left.\sum_{i=1}^{h} \rho_{i}\left(\theta_{k-1}\right)\left(\left(I-\Lambda_{i} C\right) N F H_{d}\right) \quad \sum_{i=1}^{h} \rho_{i}\left(\theta_{k-1}\right) \Lambda_{i} F H_{d}\right] .
\end{aligned}
$$

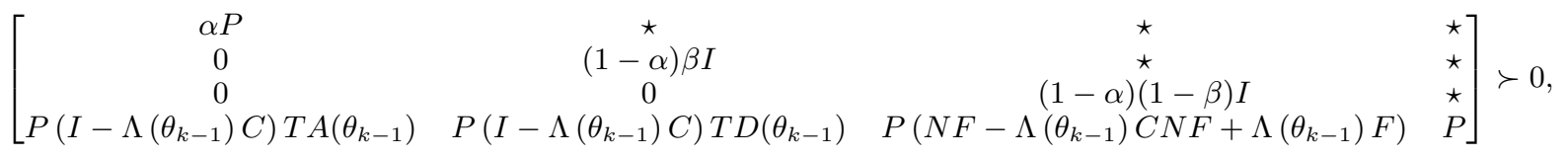

intersection zonotope with time should be limited. To achieve this, we use the reduction operator $\downarrow_{q, W}(\cdot)$ to fix the maximum number of columns of the intersection zonotope to preserve the inclusion property:

$$
\left\langle\hat{p}_{k-1}, \hat{H}_{k-1}\right\rangle \subseteq\left\langle\hat{p}_{k-1}, \bar{H}_{k-1}\right\rangle
$$

with $\bar{H}_{k-1}=\downarrow_{q, W}\left(\hat{H}_{k-1}\right)$, where $q$ is maximum column of $\bar{H}_{k-1}$ and $W$ denotes a weighting matrix of appropriate dimension.

Considering the polytopic form of the system (1), we introduce the polytopic representation of the parameterized intersection zonotope in the following corollary.

Corollary 1. Consider the descriptor LPV system (1). If there exists a parameter-varying correction matrix $\Lambda\left(\theta_{k-1}\right)$ in the following polytopic form:

$$
\Lambda\left(\theta_{k-1}\right)=\sum_{i=1}^{h} \rho_{i}\left(\theta_{k-1}\right) \Lambda_{i}
$$

with $\Lambda_{i} \in \mathbb{R}^{n_{x} \times n_{y}}, i=1, \ldots, h$, then the intersection zonotope $\hat{\mathcal{X}}_{k}\left(\Lambda\left(\theta_{k-1}\right)\right)$ can be reformulated in (15).

Proof. Based on (2), $A\left(\theta_{k-1}\right), B\left(\theta_{k-1}\right)$ and $D\left(\theta_{k-1}\right)$ can be reformulated by

$$
\left[\begin{array}{l}
A\left(\theta_{k-1}\right) \\
B\left(\theta_{k-1}\right) \\
D\left(\theta_{k-1}\right)
\end{array}\right]=\sum_{j=1}^{h} \rho_{j}\left(\theta_{k-1}\right)\left[\begin{array}{c}
A_{j} \\
B_{j} \\
D_{j}
\end{array}\right], j=1, \ldots, h,
$$

with $\rho_{j}\left(\theta_{k-1}\right) \geq 0$ and $\sum_{j=1}^{h} \rho_{j}\left(\theta_{k-1}\right)=1$. By combining (9) with (14) and (16), we obtain (15).

\section{B. Optimal Correction Matrix}

Since all the uncertain states are bounded in the intersection zonotope $\hat{\mathcal{X}}_{k}\left(\Lambda\left(\theta_{k-1}\right)\right)$, we would like to find a suitable correction matrix $\Lambda\left(\theta_{k-1}\right)$ in such a way that the size of $\hat{\mathcal{X}}_{k}\left(\Lambda\left(\theta_{k-1}\right)\right)$ is limited. We first introduce two definitions for measuring the size of a zonotope.

Definition 5. Given $\mathcal{Z}=\langle p, H\rangle \subseteq \mathbb{R}^{n}$, the radius is defined by

$$
\ell=\max _{z \in \mathcal{Z}}\|z-p\|^{2}=\max _{b \in \mathbf{B}^{r}}\|H b\|^{2}
$$

Definition 6. Given $\mathcal{Z}=\langle p, H\rangle \subseteq \mathbb{R}^{n}$, the $P$-radius is defined by

$$
\varrho=\max _{z \in \mathcal{Z}}\|z-p\|_{P}^{2}=\max _{b \in \mathbf{B}^{r}}\|H b\|_{P}^{2},
$$

with $P \in \mathbb{R}^{n \times n}$ and $P=P^{\top} \succ 0$.

Based on these definitions, we propose the condition to limit the size of $\hat{\mathcal{X}}_{k}\left(\Lambda\left(\theta_{k-1}\right)\right)$ in the following theorem.

Theorem 2. Consider the descriptor LPV system (1) and $\hat{\mathcal{X}}_{k}\left(\Lambda\left(\theta_{k-1}\right)\right)$ in (9). If there exists a matrix $P \in \mathbb{R}^{n_{x} \times n_{x}}$ with $P=P^{\top} \succ 0$, a parameter-varying correction matrix $\Lambda\left(\theta_{k-1}\right) \in \mathbb{R}^{n_{x} \times n_{y}}$, and a positive scalar $\gamma>0$ such that (19) and

$$
\left[\begin{array}{cc}
I & \star \\
\gamma P & P
\end{array}\right] \preceq 0
$$

then the parameterized intersection zonotope $\hat{\mathcal{X}}_{k}\left(\Lambda\left(\theta_{k-1}\right)\right)$, $\forall k \in \mathbb{N}_{+}$satisfies

$$
\begin{aligned}
& \varrho_{k} \leq \alpha \varrho_{k-1}+(1-\alpha) \ell_{d} \\
& \ell_{k} \leq \gamma^{2} \varrho_{k}
\end{aligned}
$$

where $\alpha, \beta \in(0,1)$ and

$$
\ell_{d}=\max _{b_{1} \in \mathbf{B}^{n_{d}}} \beta\left\|H_{d} b_{1}\right\|^{2}+\max _{b_{2} \in \mathbf{B}^{n_{d}}}(1-\beta)\left\|H_{d} b_{2}\right\|^{2} .
$$

Proof. From Definition 6, the $P$-radius of the intersection zonotope $\hat{\mathcal{X}}_{k}\left(\Lambda\left(\theta_{k-1}\right)\right)$ in (9) at time instant $k$ can be formulated as

$$
\varrho_{k}=\max _{\hat{z} \in \mathbf{B}^{n+2 n_{d}}}\left\|\hat{H}_{k}\left(\Lambda\left(\theta_{k-1}\right)\right) \hat{z}\right\|_{P}^{2},
$$

where $\hat{z} \in \mathbf{B}^{n+2 n_{d}}$ is an unitary vector. According to (10b), the vector $\hat{z}$ can be partitioned to be $\hat{z}=\left[\begin{array}{lll}\bar{z}^{\top} & b_{1}^{\top} & b_{2}^{\top}\end{array}\right]^{\top}$ with $\bar{z} \in \mathbf{B}^{n}$. By combining (21a) and (22), we obtain that

$$
\begin{aligned}
& \max _{\hat{z} \in \mathbf{B}^{n+2 n_{d}}}\left\|\hat{H}_{k}\left(\Lambda\left(\theta_{k-1}\right)\right) \hat{z}\right\|_{P}^{2} \leq \max _{\bar{z} \in \mathbf{B}^{n}} \alpha\left\|\hat{H}_{k-1} \bar{z}\right\|_{P}^{2} \\
& \quad+\max _{b_{1} \in \mathbf{B}^{n_{d}}}(1-\alpha) \beta\left\|H_{d} b_{1}\right\|^{2} \\
& \quad+\max _{b_{2} \in \mathbf{B}^{n_{d}}}(1-\alpha)(1-\beta)\left\|H_{d} b_{2}\right\|^{2}
\end{aligned}
$$




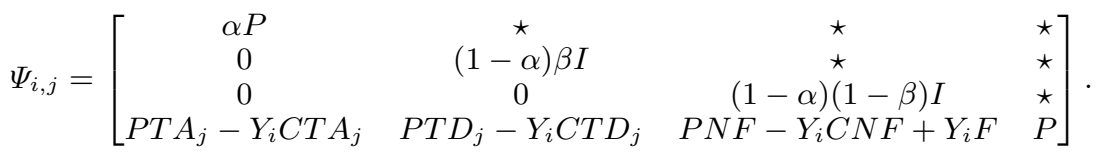

Therefore, we obtain a sufficient condition of (23) as

$$
\begin{aligned}
& \hat{z}^{\top} \hat{H}_{k}\left(\Lambda\left(\theta_{k-1}\right)\right)^{\top} P \hat{H}_{k}\left(\Lambda\left(\theta_{k-1}\right)\right) \hat{z} \\
& \quad-\alpha \bar{z}^{\top} \hat{H}_{k-1}^{\top} P \hat{H}_{k-1} \bar{z}-(1-\alpha) \beta b_{1}^{\top} H_{d}^{\top} H_{d} b_{1} \\
& \quad-(1-\alpha)(1-\beta) b_{2}^{\top} H_{d}^{\top} H_{d} b_{2}<0,
\end{aligned}
$$

for $\forall \hat{z}, \forall \bar{z}, \forall b_{1}$ and $\forall b_{2}$. Set $\xi=\hat{H}_{k-1} \bar{z}, \phi=H_{d} b_{1}$ and $\varphi=$ $H_{d} b_{2}$. By substituting $\hat{H}_{k}\left(\Lambda\left(\theta_{k-1}\right)\right)$ defined (10b) in (24), it follows that

$$
\left[\begin{array}{l}
\xi \\
\phi \\
\varphi
\end{array}\right]^{\top} \underbrace{\left[\begin{array}{ccc}
\Omega_{11} & \star & \star \\
\Omega_{21} & \Omega_{22} & \star \\
\Omega_{31} & \Omega_{32} & \Omega_{33}
\end{array}\right]}_{\Omega}\left[\begin{array}{l}
\xi \\
\phi \\
\varphi
\end{array}\right]<0
$$

with

$$
\begin{aligned}
\Omega_{11}= & A\left(\theta_{k-1}\right)^{\top} T^{\top}\left(I-\Lambda\left(\theta_{k-1}\right) C\right)^{\top} P\left(I-\Lambda\left(\theta_{k-1}\right) C\right) T A\left(\theta_{k-1}\right) \\
& -\alpha P, \\
\Omega_{21}= & D\left(\theta_{k-1}\right)^{\top} T^{\top}\left(I-\Lambda\left(\theta_{k-1}\right) C\right)^{\top} P\left(I-\Lambda\left(\theta_{k-1}\right) C\right) T A\left(\theta_{k-1}\right), \\
\Omega_{22}= & D\left(\theta_{k-1}\right)^{\top} T^{\top}\left(I-\Lambda\left(\theta_{k-1}\right) C\right)^{\top} P\left(I-\Lambda\left(\theta_{k-1}\right) C\right) T D\left(\theta_{k-1}\right) \\
& \quad-(1-\alpha) \beta I, \\
\Omega_{31}= & \left(N F-\Lambda\left(\theta_{k-1}\right) C N F+\Lambda\left(\theta_{k-1}\right) F\right)^{\top} P\left(I-\Lambda\left(\theta_{k-1}\right) C\right) T A\left(\theta_{k-1}\right), \\
\Omega_{32}= & \left(N F-\Lambda\left(\theta_{k-1}\right) C N F+\Lambda\left(\theta_{k-1}\right) F\right)^{\top} P\left(I-\Lambda\left(\theta_{k-1}\right) C\right) T D\left(\theta_{k-1}\right), \\
\Omega_{33}= & \left(N F-\Lambda\left(\theta_{k-1}\right) C N F+\Lambda\left(\theta_{k-1}\right) F\right)^{\top} P\left(N F-\Lambda\left(\theta_{k-1}\right) C N F+\Lambda\left(\theta_{k-1}\right) F\right) \\
& \quad-(1-\alpha)(1-\beta) I .
\end{aligned}
$$

By the definition of a positive definite matrix, (25) implies $\Omega \prec 0$. By applying the Schur complement lemma [22] to this matrix inequality, we obtain (19).

On the other hand, by Definition 5 , the radius of the intersection zonotope $\hat{\mathcal{X}}_{k}\left(\Lambda\left(\theta_{k-1}\right)\right)$ in (9) at time instant $k$ can be formulated as $\ell_{k}=\max _{\hat{z} \in \mathbf{B}^{n+2 n_{d}}}\left\|\hat{H}_{k}\left(\Lambda\left(\theta_{k-1}\right)\right) \hat{z}\right\|^{2}$. From (21b), we derive

$$
I-\gamma^{2} P \preceq 0 .
$$

By applying the Schur complement lemma to (26), we thus obtain (20).

Considering that $A\left(\theta_{k-1}\right)$ and $\Lambda\left(\theta_{k-1}\right)$ are defined in the polytopic form, (19) leads to a double sum problem. The following result is used for the reformulation of a double sum problem.

Lemma 2 ([23], [24]). Consider the following double-sum condition

$$
\Gamma\left(\vartheta_{k}, \vartheta_{k}\right)=\sum_{i=1}^{r} \sum_{j=1}^{r} \mu_{i}\left(\vartheta_{k}\right) \mu_{j}\left(\vartheta_{k}\right) \Gamma_{i, j} \succ 0 .
$$

Then, the condition (27) is fulfilled provided that the following conditions hold:

$$
\begin{aligned}
& \Gamma_{i, i} \succ 0, \quad i=1, \ldots, r \\
& \frac{2}{r-1} \Gamma_{i, i}+\Gamma_{i, j}+\Gamma_{j, i} \succeq 0, \quad, 1 \leq i<j \leq r .
\end{aligned}
$$

Based on Lemma 2, we now reformulate (19) with multiple vertices in the form of (27) in the following corollary.

Corollary 2. Consider the descriptor LPV system (1). If there exist matrices $P \in \mathbb{R}^{n_{x} \times n_{x}}$ with $P=P^{\top} \succ 0$ and $Y_{i} \in$ $\mathbb{R}^{n_{x} \times n_{y}}$ for $i=1, \ldots, h$ such that

$$
\begin{aligned}
& \Psi_{i, i} \succ 0, \quad i=1, \ldots, h, \\
& \frac{2}{h-1} \Psi_{i, i}+\Psi_{i, j}+\Psi_{j, i} \succeq 0, \quad, 1 \leq i<j \leq h,
\end{aligned}
$$

with $\Psi_{i, j}$ as in (30), then (19) is satisfied.

Proof. For the polytopic representation of $A\left(\theta_{k-1}\right), D\left(\theta_{k-1}\right)$ and $\Lambda\left(\theta_{k-1}\right)$, (19) can be reformulated in a double-sum form as (27). Thus, we obtain (29) by means of (28).

Based on the condition in Theorem 2, an adaptive bound, that is the upper bound of the radius of the intersection zonotope, can be obtained in the following theorem.

Theorem 3. The $\mathcal{L}_{\infty}$ performance of the radius of the intersection zonotope $\hat{\mathcal{X}}_{k}\left(\Lambda\left(\theta_{k-1}\right)\right)$ in (9) at time instant $k$ is characterized by

$$
\ell_{k} \leq \gamma^{2} \alpha^{k} \varrho_{0}+\gamma^{2}\|d\|_{\infty}^{2}
$$

with $\varrho_{0}=\max _{b_{0} \in \mathbf{B}^{n_{0}}}\left\|H_{0} b_{0}\right\|_{P}^{2}$.

Proof. From (3) and (22), we have $\ell_{d}=\max _{b \in \mathbf{B}^{n_{d}}}\left\|H_{d} b\right\|^{2}=$ $\|d\|_{\infty}^{2}$. From (21a), for some $\alpha \in(0,1)$, we can derive

$$
\begin{aligned}
\varrho_{k} & \leq \alpha \varrho_{k-1}+(1-\alpha)\|d\|_{\infty}^{2}, \\
& \leq \alpha^{k} \varrho_{0}+(1-\alpha) \sum_{i=0}^{k-1} \alpha^{i}\|d\|_{\infty}^{2}, \\
& \leq \alpha^{k} \varrho_{0}+\|d\|_{\infty}^{2} .
\end{aligned}
$$

Therefore, from (21b), we obtain

$$
\ell_{k} \leq \gamma^{2} \varrho_{k} \leq \gamma^{2}\left(\alpha^{k} \varrho_{0}+\|d\|_{\infty}^{2}\right)
$$

which gives (31).

Remark 3. Note that Theorem 2 provides a procedure to obtain the most adjusted zonotope that outer-bounds the intersection of the measurement consistent state set $\overline{\mathcal{X}}_{k}$ and the consistent uncertain state set $\mathcal{X}_{y_{k}}$. The radius $\ell_{k}$ (introduced in Definition 5) is used to measure the size of the resulting zonotope. According to Theorem 2, this radius satisfies (21b). On the other hand, Theorem 3 introduces a time-varying bound 
for this radius considering the worst-case disturbances. As shown in the proof of Theorem 3, the relation of this worstcase bound with the one obtained in Theorem 2 is given by (32) which leads to (31). This inequality establishes that the timevarying radius $\ell_{k}$ is bounded by $\varrho_{0}$ (from the initial condition), the worst-case disturbance, a given scalar $\alpha \in(0,1)$ as well as a scalar $\gamma>0$. As the time $k$ increases, the term $\alpha^{k}$ is going to be zero. Hence, for $k \geq k_{M}$ (let us denote $k_{M}$ as an arbitrary large integer), a worst-case bound for $\ell_{k}$ is obtained considering the worst-case disturbance as $\ell_{k} \leq \gamma^{2}\|d\|_{\infty}^{2}$ for $\gamma>0$.

Based on Remark 3, the optimal polytopic correction matrices $\Lambda_{i}$ for $i=1, \ldots, h$ can be found by solving the following optimization problem:

$$
\min _{P, Y_{i}} \gamma
$$

subject to (20) and (29), which produces the least conservative worst case bound of $\ell_{k}$ can be obtained.

Then, the optimal solutions of the optimization problem (33) give $\Lambda_{i}^{*}=P^{*-1} Y_{i}^{*}$ for $i=1, \ldots, h$.

Remark 4. The constraints in (29) are linear and hence convex with given $\alpha, \beta \in(0,1)$. To deal with term $\gamma P$ in (20), the optimization problem (33) can be solved by a linear programming solver with a line search to find the minimum $\gamma$.

Remark 5. The condition (21a) can be replaced by (9) in [25], which can be formulated as

$$
\varrho_{k} \leq \sigma \varrho_{k-1}+\epsilon
$$

with $\sigma \in[0,1)$ and $\epsilon$ is a scalar that can be determined by system uncertainties. From $d_{k}=\left[\begin{array}{ll}w_{k}^{\top} & v_{k}^{\top}\end{array}\right]^{\top} \in \mathcal{D}$, $\forall k \in \mathbb{N}$, we consider that the set $\mathcal{D}$ can be rewritten by the Cartesian product as $\mathcal{D}=\mathcal{W} \times \mathcal{V}$ with $w_{k} \in \mathcal{W}=\left\langle 0, H_{w}\right\rangle$ and $v_{k} \in \mathcal{V}=\left\langle 0, H_{v}\right\rangle, \forall k \in \mathbb{N}$, where $H_{w}$ and $H_{v}$ are the segment matrices of appropriate dimensions. Therefore, according to [25], $\epsilon$ can be estimated by

$\epsilon=\max _{\bar{b}_{1} \in \mathbf{B}^{n_{w}}}\left\|H_{w} \bar{b}_{1}\right\|^{2}+\max _{\bar{b}_{2} \in \mathbf{B}^{n_{v}}}\left\|H_{v} \bar{b}_{2}\right\|^{2}+\max _{\bar{b}_{3} \in \mathbf{B}^{n_{v}}}\left\|H_{v} \bar{b}_{3}\right\|^{2}$.

From (34), we follow the proof of Theorem 2 to obtain (35), which can also be reformulated to be the polytopic form as presented in Corollary 2.

Besides, when time tends to infinity, (34) can be bounded by $\varrho_{\infty} \leq \sigma \varrho_{\infty}+\epsilon$ leading to $\varrho_{\infty} \leq \frac{\epsilon}{1-\sigma}$. Based on [25, Algorithm 2], to minimize the P-radius $\varrho_{\infty}$ of the intersection zonotope (9), we can solve an eigenvalue optimization problem with a scalar $\tau>0$ as follows:

$$
\max _{P, Y_{i}} \tau
$$

subject to $\frac{(1-\sigma) P}{\epsilon} \succeq \tau I$ and the polytopic form of (35).

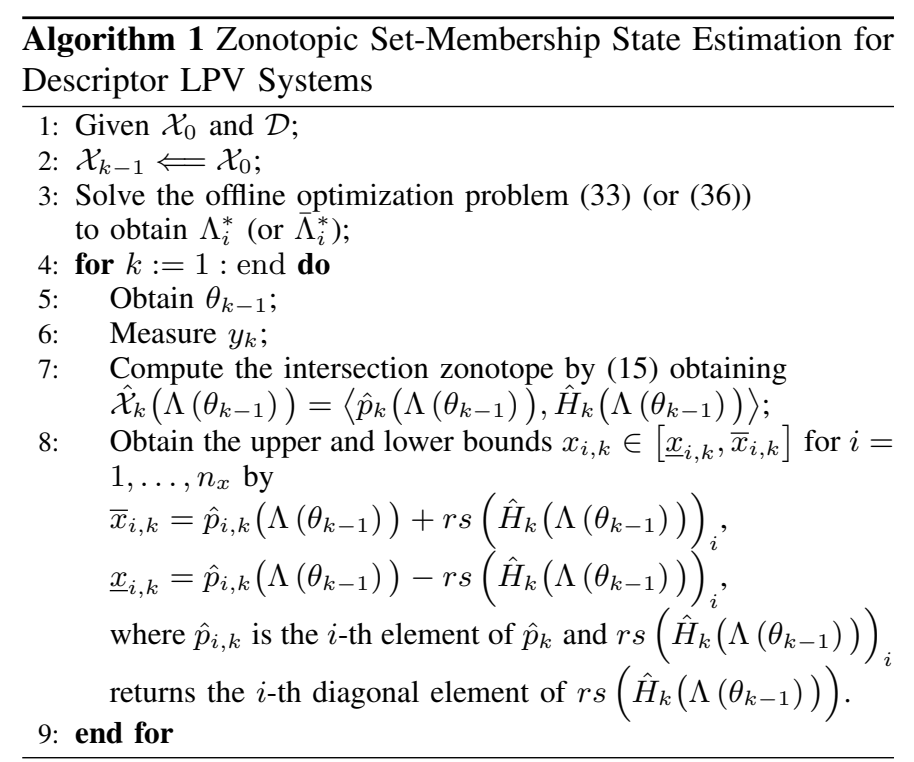

Then, the optimal solutions of (36) give $\bar{\Lambda}_{i}^{*}=P^{*-1} Y_{i}^{*}$ for $i=1, \ldots, h$.

Remark 6. The main difference between using criteria (21a) and (34) is that although the resulting approaches compute the intersection zonotope based on the same structure in Corollary 1, the corresponding correction matrices $\Lambda_{i}^{*}$ and $\bar{\Lambda}_{i}^{*}$ for $i=1, \ldots, h$ are obtained using different objectives. In the case of the approach based on (21a) proposed in this paper, the optimization problem (33) seeks to minimize the upper bound of the time-varying radius (based on Definition 4) of the intersection zonotope, while in the approach based on (34), the optimization problem (36) minimizes the steady $P$-radius of the intersection zonotope.

Finally, we summarize the proposed set-membership state estimation approach for the discrete-time descriptor LPV system (1) in Algorithm 1.

\section{CAse Study}

\section{A. The Truck-Trailer Model}

From [19], the truck-trailer system is modeled by (1) in the polytopic form as in Section II, where

$$
\begin{aligned}
& E=\left[\begin{array}{cccc}
1 & 0 & 0 & 0 \\
0 & 1 & 0 & 0 \\
0 & 0 & 1 & -1 \\
0 & 0 & 0 & 0
\end{array}\right], A_{1}=\left[\begin{array}{cccc}
1.025 & 0 & 0 & 0 \\
-0.218 & 1 & 0 & 0 \\
0 & 0 & 1 & 1 \\
0 & 0.06 & 0 & 1
\end{array}\right], \\
& A_{2}=\left[\begin{array}{cccc}
1.05 & 0 & 0 & 0 \\
-0.436 & 1 & 0 & 0 \\
0 & 0 & 1 & 1 \\
0 & 0.12 & 0 & 1
\end{array}\right], B_{1}=\left[\begin{array}{c}
-0.025 \\
0 \\
0 \\
0
\end{array}\right], B_{2}=\left[\begin{array}{c}
-0.05 \\
0 \\
0 \\
0
\end{array}\right] \text {, } \\
& D_{1}=\left[\begin{array}{cccc}
0 & 0 & 0 & 0 \\
0 & 0 & 0 & 0 \\
-0.12 & 0 & 0 & 0 \\
0 & 0 & 0 & 0
\end{array}\right], D_{2}=\left[\begin{array}{cccc}
0 & 0 & 0 & 0 \\
0 & 0 & 0 & 0 \\
-0.24 & 0 & 0 & 0 \\
0 & 0 & 0 & 0
\end{array}\right] \text {, }
\end{aligned}
$$

and the sampling time is $\Delta t=0.2 s$. The speed of backing up $\theta_{k}$ varies in the range $\theta_{k} \in[-1.2,-0.6]$ as presented in 


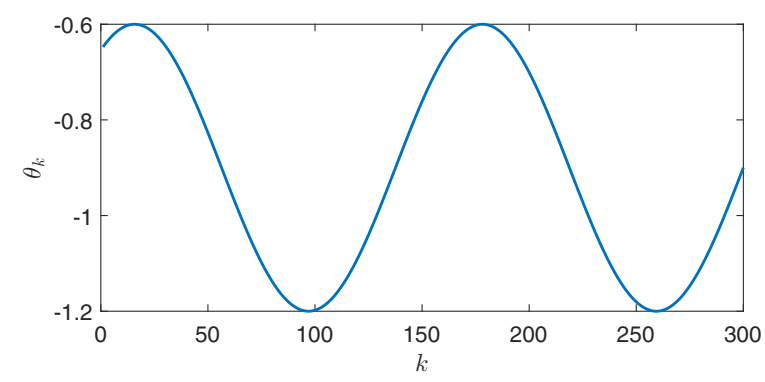

Figure 1. The time-varying parameter $\theta_{k}$.

Figure 1 and the weighting functions $\rho_{j}\left(\theta_{k}\right)$ for $j=1,2$ are computed as $\rho_{1}\left(\theta_{k}\right)=\frac{\theta_{k}+1.2}{0.6}$ and $\rho_{2}\left(\theta_{k}\right)=\frac{\theta_{k}+0.6}{-0.6}$. Besides, the initial state is chosen as $x_{0}=\left[\begin{array}{llll}0.1745 & 0.3491 & 3 & -0.4189\end{array}\right]^{\top}$ and the initial estimation is bounded in the zonotope $\mathcal{X}_{0}=$ $\left\langle p_{0}, H_{0}\right\rangle$ with $p_{0}=x_{0}$ and $H_{0}=\operatorname{diag}\left(\left[\begin{array}{llll}0.02 & 0.02 & 0.1 & 0.02\end{array}\right]\right)$.

Besides, to reduce the computation time and limit the growing complexity of the resulting zonotope, we set $q=$ 20 in the zonotope reduction operator $\downarrow_{q, W}(H)$ and the weighting matrix is chosen as $W=P . d_{k} \in \mathcal{D}, \forall k \in \mathbb{N}$ with $H_{d}=\operatorname{diag}\left(\left[\begin{array}{llll}0.03 & 0.004 & 0.004 & 0.004\end{array}\right]\right)$.

From Lemma 1 , since $S$ is an arbitrary matrix in (5), we

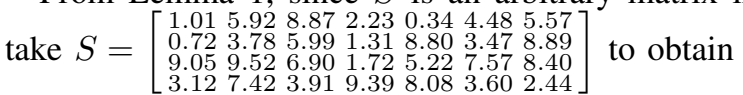

$T=\left[\begin{array}{rrrr}1 & 2.7864 & 3.3204 & 2.2327 \\ 0 & -2.0097 & 3.8036 & 1.3126 \\ 0 & 2.1462 & 2.9088 & 1.7248 \\ 0 & -0.3293 & 0.5831 & 9.3860\end{array}\right], N=\left[\begin{array}{ccc}-2.7864 & -3.3204 & 3.3204 \\ 3.0097 & -3.8036 & 3.8036 \\ -2.1462 & -1.9088 & 2.9088 \\ 0.3293 & -0.58831 & 1.5831\end{array}\right]$.

From (21a), the convergence rate of the $P$-radius $\varrho_{k}$ is described by $\alpha$. By simulations, we tune $\alpha \in(0,1)$ to find a minimum $\gamma$. Moreover, we choose $\beta=0.5$ considering the worst-case uncertainties in two equivalent parts.

\section{B. Results}

The optimization problem (33) is solved using the YALMIP toolbox [26] and the MOSEK solver [27]. All the simulations are carried out in a PC with CPU of Intel (R) Core (TM) i7-5500U $2.4 \mathrm{GHz}$ and $12 \mathrm{~GB}$ memory. By means of a line search, we obtain the minimum $\gamma=11.95$ with $\alpha=0.75$ and the optimal polytopic correction matrices $\Lambda_{i}^{*}, i=1,2$,

$\Lambda_{1}^{*}=\left[\begin{array}{ccc}0.8359 & -0.0031 & 0.1491 \\ 1.0755 & -0.1189 & 0.0239 \\ -0.0558 & 0.9437 & 0.0723 \\ 0.0496 & 0.0466 & 0.9378\end{array}\right], \Lambda_{2}^{*}=\left[\begin{array}{ccc}0.7413 & 0.1768 & 0.0952 \\ 1.0728 & -0.1191 & 0.0274 \\ -0.0547 & 0.9386 & 0.0743 \\ 0.0464 & 0.0519 & 0.9371\end{array}\right]$

As a comparison, we also solve the optimization problem (36) by a line search with $\sigma \in[0,1)$ using [25, Algorithm 2]. Then, we obtain the maximum $\tau=0.00024$ with $\sigma=0.8$ and the polytopic correction matrices $\bar{\Lambda}_{i}^{*}$ for $i=1,2$,

$\bar{\Lambda}_{1}^{*}=\left[\begin{array}{ccc}1.8397 & -2.3403 & 0.8747 \\ 0.9557 & 0.0102 & 0.0185 \\ 0.0098 & 0.9453 & 0.0222 \\ 0.0200 & 0.0223 & 0.9730\end{array}\right], \bar{\Lambda}_{2}^{*}=\left[\begin{array}{ccc}1.9712 & -2.4276 & 0.8667 \\ 0.9646 & -0.0022 & 0.0199 \\ -0.0020 & 0.9627 & 0.0196 \\ 0.0206 & 0.0203 & 0.9740\end{array}\right]$.

By implementing Algorithm 1 for $N=300$ sampling steps with $\Lambda_{i}^{*}$ and $\bar{\Lambda}_{i}^{*}$ separately, the comparison results of the state estimation are shown in Figure 2 and Figure 3, where real states are plotted by red stars as the validation. From these two figures, based on the structure of the intersection

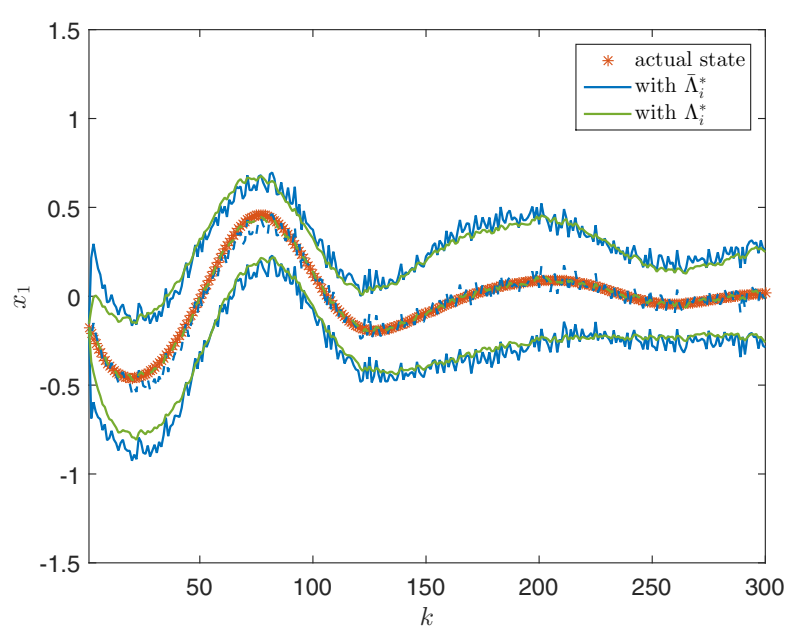

Figure 2. The state estimation result of $x_{1}$.

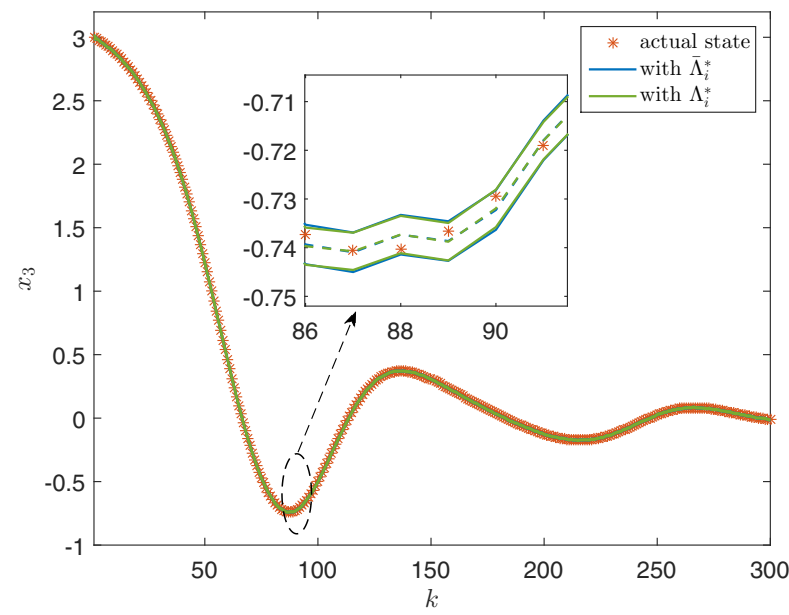

Figure 3. The state estimation result of $x_{3}$.

zonotope in Theorem 1, the proposed approach with $\Lambda_{i}^{*}$ and the comparison approach with $\bar{\Lambda}_{i}^{*}$ are able to estimate uncertain states in dash lines and propagate the estimation interval in solid lines (green ones for $\Lambda_{i}^{*}$ and blue ones for $\bar{\Lambda}_{i}^{*}$ ).

In order to quantitatively compare the results with $\Lambda_{i}^{*}$ and $\bar{\Lambda}_{i}^{*}$, we define the state estimation error between the estimated states and real states as $e_{k}=x_{k}-\hat{p}_{k}$ and the mean square error $(M S E)$ by $M S E=\frac{1}{N} \sum_{k=1}^{N} \frac{1}{n_{x}}\left\|e_{k}\right\|^{2}$. Since system uncertainties are propagated to the states during iterations, we also measure $\operatorname{rs}\left(\hat{H}_{k}\right)$ to compare the size of the intersection zonotope with $\Lambda_{i}^{*}$ and $\bar{\Lambda}_{i}^{*}$ bounding uncertain states. Table I shows the comparison result of $M S E$, the root mean square of $r s\left(\hat{H}_{k}\right)$ as well as the computation time. For this case study, it is clear from this table that the estimation error of the proposed approach is smaller as well as the size of intersection zonotopes. $\Lambda_{i}^{*}$ and $\bar{\Lambda}_{i}^{*}$ are obtained from offline optimizations while the state estimation results are from online numerical computation using Corollary 1 


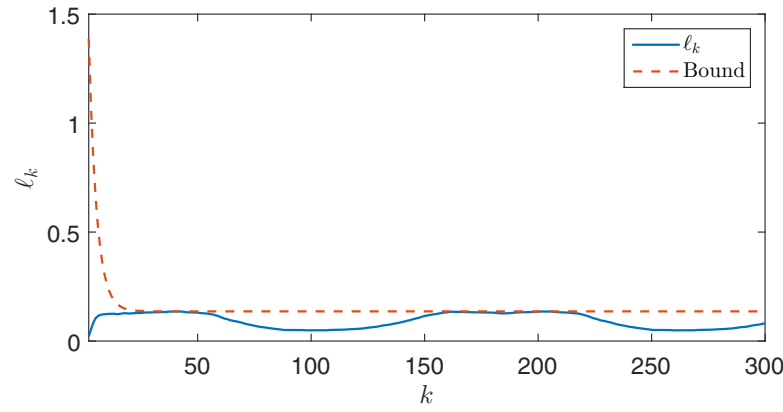

Figure 4. The $\mathcal{L}_{\infty}$ performance with $\Lambda_{i}^{*}$.

Table I

The Comparison Result Between $\Lambda_{i}^{*} \operatorname{And} \bar{\Lambda}_{i}^{*}$.

\begin{tabular}{cccc}
\hline Approach & $M S E$ & $r s(\hat{H})$ & Computation Time $[s]$ \\
\hline$\Lambda_{i}^{*}$ & $4.7362 \mathrm{e}-05$ & 0.1332 & 0.0090 \\
$\bar{\Lambda}_{i}^{*}$ & $5.2623 \mathrm{e}-04$ & 0.1459 & 0.0099 \\
\hline
\end{tabular}

(Theorem 1). As shown in Table I, the average computation time per one iteration with $\Lambda_{i}^{*}$ and $\bar{\Lambda}_{i}^{*}$ is less than $0.01 s$, which is significantly shorter than the sampling time $\Delta t=0.2$ allowing the real-time implementation.

Besides, with the proposed approach, the time-varying radius of the intersection zonotope is expected to be lower than the adaptive bound based on $\gamma$ (as presented in Theorem 3), which is called the $\mathcal{L}_{\infty}$ performance. In Figure 4, with the optimal solution $\gamma$, we can see that the radius of the intersection zonotope at each time is always constrained.

\section{CONClusion}

In this note, we have presented a zonotopic set-membership state estimation approach for discrete-time descriptor LPV systems considering unknown-but-bounded uncertainties. We have constructed the parameterized intersection zonotope to bound the set of uncertain states that are consistent with the descriptor LPV model and the measured outputs. In the proposed approach, the optimal correction matrix can be obtained by solving an offline optimization problem that minimizes a time-varying radius. Besides, the obtained estimation result is obtained in a deterministic zonotope, whose radius is bounded using the $\mathcal{L}_{\infty}$ performance index. In the truck-trailer case study, we have compared the proposed approach with the one based on an existing $P$-radius criterion. It can be concluded that in this case study, the proposed approach is able to provide more accurate state estimation results. Moreover, since only offline optimization is involved, the proposed approach can be easily implemented in real-time applications.

\section{REFERENCES}

[1] T. Alamo, J. Bravo, and E. Camacho, "Guaranteed state estimation by zonotopes," Automatica, vol. 41, no. 6, pp. 1035-1043, 2005.

[2] C. Combastel, "Zonotopes and Kalman observers: Gain optimality under distinct uncertainty paradigms and robust convergence," Automatica, vol. 55, pp. 265-273, 2015.
[3] J. Bravo, T. Alamo, and E. Camacho, "Bounded error identification of systems with time-varying parameters," IEEE Transactions on Automatic Control, vol. 51, no. 7, pp. 1144-1150, 2006.

[4] M. Kieffer and E. Walter, "Guaranteed estimation of the parameters of nonlinear continuous-time models: Contributions of interval analysis," International Journal of Adaptive Control and Signal Processing, vol. 25, no. 3, pp. 191-207, 2011.

[5] I. Fagarasan, S. Ploix, and S. Gentil, "Causal fault detection and isolation based on a set-membership approach," Automatica, vol. 40, no. 12, pp. 2099-2110, 2004.

[6] C. Jauberthie, N. Verdière, and L. Travé-Massuyès, "Fault detection and identification relying on set-membership identifiability," Annual Reviews in Control, vol. 37, no. 1, pp. 129-136, 2013.

[7] V. Puig, "Fault diagnosis and fault tolerant control using set-membership approaches: Application to real case studies," International Journal of Applied Mathematics and Computer Science, vol. 20, no. 4, pp. 619635, 2010.

[8] D. Efimov, L. Fridman, T. Raïssi, A. Zolghadri, and R. Seydou, "Interval estimation for LPV systems applying high order sliding mode techniques," Automatica, vol. 48, no. 9, pp. 2365-2371, 2012.

[9] T. Raïssi, D. Efimov, and A. Zolghadri, "Interval state estimation for a class of nonlinear systems," IEEE Transactions on Automatic Control, vol. 57, no. 1, pp. 260-265, 2012.

[10] W. Kühn, "Rigorously computed orbits of dynamical systems without the wrapping effect," Computing, vol. 61, no. 1, pp. 47-67, 1998.

[11] X. Yang and J. Scott, "A comparison of zonotope order reduction techniques," Automatica, vol. 95, pp. 378-384, 2018.

[12] L. Dai, Singular Control Systems. Berlin Heidelberg, Germany: Springer, 1989.

[13] G. Duan, Analysis and Design of Descriptor Linear Systems. New York, USA: Springer, 2010.

[14] Y. Wang, V. Puig, and G. Cembrano, "Non-linear economic model predictive control of water distribution networks," Journal of Process Control, vol. 56, pp. 23-34, 2017.

[15] B. Stevens, F. L. Lewis, and E. Johnson, Aircraft Control and Simulation: Dynamics, Controls Design, and Autonomous Systems. New York, USA: Wiley-Blackwell, 2016.

[16] L. Biegler, S. Campbell, and V. Mehrmann, Control and Optimization with Differential-Algebraic Constraints. Philadelphia, USA: Society for Industrial and Applied Mathematics, 2012.

[17] M. Althoff and B. H. Krogh, "Reachability analysis of nonlinear differential-algebraic systems," IEEE Transactions on Automatic Control, vol. 59, no. 2, pp. 371-383, 2014.

[18] I. Masubuchi, J. Kato, M. Saeki, and A. Ohara, "Gain-scheduled controller design based on descriptor representation of LPV systems: application to flight vehicle control," in IEEE Conference on Decision and Control (CDC), vol. 1, 2004, pp. 815-820.

[19] Z. Wang, M. Rodrigues, D. Theilliol, and Y. Shen, "Actuator fault estimation observer design for discrete-time linear parameter-varying descriptor systems," International Journal of Adaptive Control and Signal Processing, vol. 29, no. 2, pp. 242-258, 2015.

[20] Z. Wang, P. Shi, and C. Lim, " $H_{-} / H_{\infty}$ fault detection observer in finite frequency domain for linear parameter-varying descriptor systems," Automatica, vol. 86, pp. 38-45, 2017.

[21] H. Hamdi, M. Rodrigues, C. Mechmeche, D. Theilliol, and N. Benhadj Braiek, "Fault detection and isolation in linear parametervarying descriptor systems via proportional integral observer," International Journal of Adaptive Control and Signal Processing, vol. 26, no. 3, pp. 224-240, 2012.

[22] S. Boyd, L. El Ghaoui, E. Feron, and V. Balakrishnan, Linear Matrix Inequalities in System and Control Theory. Philadelphia, PA: SIAM, 1994.

[23] H. Tuan, P. Apkarian, T. Narikiyo, and Y. Yamamoto, "Parameterized linear matrix inequality techniques in fuzzy control system design," IEEE Transactions on Fuzzy Systems, vol. 9, no. 2, pp. 324-332, 2001.

[24] M. Chadli, A. Abdo, and S. Ding, "Fault detection filter design for discrete-time Takagi-Sugeno fuzzy system," Automatica, vol. 49, no. 7, pp. 1996-2005, 2013.

[25] V. Le, T. Alamo, E. Camacho, C. Stoica, and D. Dumur, "A new approach for guaranteed state estimation by zonotopes," in 18th IFAC World Congress, 2011, pp. 9242-9247.

[26] J. Löfberg, "YALMIP: A toolbox for modeling and optimization in MATLAB," Taipei, Taiwan, 2004. [Online]. Available: http: //users.isy.liu.se/johanl/yalmip

[27] MOSEK ApS, The MOSEK optimization toolbox for MATLAB manual. Version 7.1 (Revision 28)., 2015. [Online]. Available: http://docs.mosek.com/7.1/toolbox/index.html 